Research Article

\title{
Optimization of Building Exit Layout: Combining Exit Decisions of Evacuees
}

\author{
Guofeng Ma, Yuqi Wang $\mathbb{D}^{D}$, and Shan Jiang $\mathbb{C}$ \\ School of Economics and Management, Tongji University, Shanghai 20092, China \\ Correspondence should be addressed to Yuqi Wang; 1930354@tongji.edu.cn
}

Received 12 November 2020; Accepted 24 May 2021; Published 3 June 2021

Academic Editor: Heap-Yih (John) Chong

Copyright ( $\odot 2021$ Guofeng Ma et al. This is an open access article distributed under the Creative Commons Attribution License, which permits unrestricted use, distribution, and reproduction in any medium, provided the original work is properly cited.

\begin{abstract}
Exits are essential to the efficiency of building evacuation due to its irreplaceable function, and the layout of multiple exits has always been the key concern for architectural design. To accurately evaluate the evacuation efficiency of different multiexit layouts and optimize the design rules, a dynamic exit decision model integrating an exit selection strategy and the social force model is developed to simulate the practical evacuation. And our proposed model outperforms the original social force model in terms of evacuation efficiency. Accordingly, different layouts are analyzed for evacuation in a single room with two exits. The analysis results reveal that evacuation time will be improved with the changes of exit locations and two parallel exits are validated as the most efficient layout among the three common categories. Affected by walking time and queuing time of evacuees, it is not conducive to evacuation whether the separation of two exits is too large or too small. Furthermore, an even symmetry is found more efficient than an asymmetric distribution of exits under some conditions. This work provides a basis for architectural designs of multiple exits and a foundation for further study of evacuation simulation.
\end{abstract}

\section{Introduction}

Evacuation describes a certain collective behavior where pedestrians are brought together in groups and move like a whole to a safe area once a danger is recognized $[1,2]$. Pedestrian evacuation has become a widely studied topic in the last few decades, and there are numerous interesting phenomena of pedestrian flow, such as oscillations [3], arching [4], the "faster-is-slower" effect [4], herding behavior [5], and "freezing-by-heating" effect [6]. In emergencies, pedestrians were observed being jammed at exits [7] and massive congestion resulted in disasters and casualties $[4,8,9]$. Uncontrolled collective behaviors and inefficient architectural designs have been identified as two key factors that contribute to tragedies [10]. Therefore, it is necessary to understand the behaviors of people in evacuation processes and improve evacuation efficiency by optimizing architectural designs [11]. Due to the actual growing complexity of the architectural designs, it is difficult for building codes to synchronize with building requirements. People have been adopting performance-based solutions to address complex design issues
$[12,13]$, and crowd evacuation simulation is viewed as an effective method to help engineers identify design shortcomings and improve building performance. Crowd modeling has emerged from a need for pedestrian simulation that adapts to technological development and challenges of laboratory experiments, and many simulations have been conducted to reproduce crowd behaviors at egress [14].

Through an extensive search of related research, three main evacuation optimization methods are recognized as follows [15]: (a) architectural design and infrastructure adjustment; (b) optimization of path planning; (c) behavioral modification and active guidance. Specifically, the architectural design of exits, considering exits are the main means of evacuation [2], is one of the problems attracting considerable attention before design efficient evacuation plans. Although some researchers have pointed out that exit layout has a significant impact on crowd behavior [16, 17], few of them examined this influence and its impact on the evacuation process [2].

The exit decisions, which reflect the multiattribute tradeoffs of evacuees between social interaction and physical 
factors [18], are bounded by available contextual information at exits, such as distance and visibility of exits, relative queue size at exits, social influence of neighbors, inertia (for maintaining initial choices), and crowd size [19, 20]. Evacuees in emergency evacuations have the motivation and ability to adjust their decisions in response to the changes in their surroundings to ensure that they make the best choice. The most basic principle is the "least effort" [21]. During the process of evacuation, as several exits appear within the perception of the fleeing pedestrians, they will pay attention to each exit and lead their ways to the exit which takes the least estimated evacuation time at any time. As the main attention of most pedestrian models is currently paid to crowd movement, the questions of exit decision and decision adaption have practical implications from the crowd modeling perspectives. There is still a need of incorporating individual decision and environmental disturbance in the behavioral level of evacuees that help involve more real aspects of evacuation management and exit designs, such as the layout of multiple exits.

This paper deals with the layout optimization problem of multiple exits in general evacuations by establishing a dynamic exit decision model that integrates an exit selection strategy and the social force model to simulate the behavior of evacuees. The abstract model which is less constrained by application scenarios will meet a variety of analysis needs. The strategy for exit selection is introduced by comparing the combination of estimated individual walking time and estimated queuing time. The approach provides simulated individuals with the ability to assess their environment (by predicting crowds at exits) and choose the most suitable destination. The model shows a more realistic evacuation compared with the original social force model. By performing simulations, the model can be used to study the relationship between exit layout and evacuation efficiency quantitatively and the simulation results can provide new insight into architectural designs.

In this paper, the earlier work related to exit optimization, crowd modeling, and exit choice is studied and discussed in Section 2. Section 3 presents a dynamic exit decision model that incorporating an exit selection strategy into the original social force model to simulate evacuees in evacuations. Section 4 compares the results of two experiments involved the two models in evacuation and confirms the accuracy of the dynamic exit decision model. The layout optimization of two exits is quantitatively analyzed in Section 5. Finally, Section 6 concludes and discusses directions for future research.

\section{Related Works}

2.1. Design of Building Exits. Building evacuation is a complex and comprehensive system including human behaviors and environmental facilities. In addition to standardizing and guiding crowd behaviors [22], performancebased architectural design is a key approach to promote efficiency and safety of evacuation [23]. During the evacuation process, exits are the main way and important design factor to ensure the safety of persons in buildings [24], and the building exits should be designed optimally to maximize evacuation efficiency. Besides the evacuation time [2], the average distance of evacuation [25], exit utilization rate [26], and the relative distance between exits (RDBE) [12] have proven to be a metric for measuring evacuation efficiency.

Although extracting trajectories of pedestrians from videos of control experiments [27] is a source of evacuation data, it is tedious and accidental. Therefore, researchers prefer to build crowd models based on the behavior logic of evacuees and repeat simulations by using the Monte Carlo technique to capture uncertainty [28]. Shin et al. [29] developed four types of mathematical models from the discrete-time dynamic network flow model to plan optimal routes for evacuees and responders. Bina and Moghadas [28] presented a framework to analyze the impact of different architectural designs on evacuation via building information modeling (BIM) in a dynamic computational model (AnyLogic code). Braga et al. [25] proposed a pathfinder type algorithm to study the relationship between location, quantity, and size of the exits and the value of the average distances.

The architectural performance designs of exits mainly focus on three aspects: shape, structure, and layout. The changing shape of exits means that parts of the structure move without affecting the structural integrity [1]. Johnson et al. [1] simulated the evacuation of agents using discrete dynamic simulation, and various adaptive geometric configurations of exit were analyzed for evaluating design optimization. Liao et al. [30] used a modified cellular automaton model to investigate the evacuation efficiencies of parallel, convex, and concave exit. It was found that in high-density situations, the concave exit gives the maximum flow rate while the convex one gives the minimum. And the concave exit leads the least competition. $\mathrm{Mu}$ et al. [31] described a wedge-shaped design for building bottleneck spaces and proved that evacuation time is reduced with this design compared to a rectangular design through the social force model. The structure of exits includes width, height, thickness, the material, and opening angles of doors. Li and $\mathrm{Xu}$ [32] analyzed the effect of exit width and door opening in buildings on the basis of a cellular automata model under different exit schemes. And individual optimal effect of the evacuated crowd was calculated as well. Arteaga and Park [23] performed a systematic evaluation of three important building design parameters (exit width, door width, and hall width) based on the social force model. Most existing works on exit layout are confined to fixed locations or satisfactory solutions from limited schemes. And limited by the application scenario [26], the conclusions may be too specific. Therefore, researchers are seeking universal solutions actively. According to design specifications and building structures, Gao et al. [13] proposed a constraint-based model to generate the optimal door positions which minimize evacuation distance. The branch and bound algorithm is used to obtain optimal combinations of positions in this model. And Khamis et al. [24] found the most optimal exit door locations by artificial bee colony (ABC) optimization algorithm and social force model became the basis for the optimizer to represent the crowd dynamics. Although the 
studies above optimize exit layout through traversal search, the main limitation is crowd modeling. The models lack considerations of evacuees' decision-making behaviors and other factors, which is potentially a crucial topic worthy of investigation.

2.2. Crowd Modeling. Due to the challenges of obtaining behavioral data from natural evacuation and crowd incidents, laboratory experiments were proposed as a solution for data collection. In addition to nonhuman organisms' experiments, some human evacuation experiments [27, 33] have performed for the study of collective behaviors. However, some conclusions may not be convincing due to realistic and psychological constraints [34]. And physiological and psychological factors that affect individual and collective behaviors are difficult to control while the chaos of experiments under panic often results in recording failure. As knowledge expands and computer technologies advance, evacuation simulation is becoming increasingly attractive. Thus, crowd modeling, as a vital part of evacuation simulation, offers operational capability in developing simulations that depend on input parameters under different types of scenarios. In general, the existing models in the literature can be divided into two classes: macroscopic models and microscopic models. Macroscopic models are top-down approaches in which the system is viewed as a holistic flow [18]. Although the models have more complete mathematical theories and computational efficiency, the algorithms are often complex. Some simplifying assumptions are made to keep theorems tractable, which might be unrealistic. Furthermore, they suffer from loss of accuracy as they are unable to describe individual behaviors and interactions [18].

Microscopic models, which mainly focus on individual behavioral characteristics, are relatively simple and straightforward, resulting in a better computational capacity. Many realistic factors can be easily incorporated into simulations, such as the individual preference [19] and impact of the surrounding environment [35]. Microscopic models are defined as bottom-up approaches where evacuees are considered as intelligent agents that have unique characteristics such as age, gender, body size, and speed [33, 36-38]. The time-space behaviors of pedestrians are described in rules or expressions involving spatial transition probabilities. In the light of the literature, there are two microscopic models most commonly used in pedestrian simulation as follows: cellular automata (CA) model and social force model.

The CA model is a typical representative of the discrete models in which pedestrians located in a lot of uniform distributed grids will take actions in accordance with certain predetermined rules by referring to the environment. Wolfram [39] defined it as a mathematical idealization of physical systems in which space and time are discrete. Although the model performs well in fast implementation and computation, there are certain limitations. First, there is a lack of attention to interactions and contacts among individuals in the model. Second, the speed and direction changing of individual movements are limited [37]. Third, it offers inaccurate results when individuals in a high-density crowd are forced into a fixed discrete cell, but large size of cells may cause a loss in computational efficiency. The social force model [13] presents pedestrian behaviors microscopically through social fields triggered by individual social behaviors, which enables researchers to explore new relations and mathematical rules of evacuee behaviors such as decision adaption. The majority of studies have investigated from an architectural optimization perspective $[33,36]$ to improve evacuation process and pedestrian decision-making with the social force model. And many efforts have been made to improve the original social force model. Besides improving the inclusivity of the model by considering different factors [37, 38], researchers expanded and improved the original model with laboratory experiments [33] or other algorithms [40, 41]. Since it is difficult to simulate real evacuation completely, there is still a lot to do with the model.

2.3. Exit Decisions of Pedestrians. Exit choice is one of the critical individual behaviors during multiexit evacuations, which leads to redistribution of the population [42] and affects the process and evacuation time [2] of emergency. In order to learn the behavior and optimize the evacuation process, researchers have devoted much effort to create and modify models for pedestrian exit choice research. Kinateder et al. [19] analyzed the effects of exit familiarity and choices of other pedestrians in an ambulatory virtual environment. The neighbor behaviors (social influence) have been proved to have a cumulative effect on individual choice, which provides evidence for the herding effect. Haghani and Sarvi [43] proposed a parsimonious discrete-choice model of decision changing considering the most influential factors that include the effects of "relative queue-size imbalance at exits," "visibility of exits," "social influence," and "inertia (for maintaining initial decisions)." Based on estimated evacuation time and shortest distance, $\mathrm{Li}$ et al. [20] proposed an estimated evacuation time model to simulate individual behaviors in multiexit case, and the choice of exits depended on pedestrian preference. Lo et al. [44] proposed an exit selection model based on game theory, in which individual choice depends on how groups of evacuees interacted. Cao et al. [35] investigated exit selection and pedestrian movement under fire emergency by using a multigrid model based on random utility theory.

In previous studies, the individual decision-making rules of evacuation models are formulated by considering various basic parameters including exit configurations [33], exit capacity [45], obstacles [46], human psychology [18], exit familiarity [19], distribution of the crowd, and environment [35]. Following the shortest time or shortest distance principle, most individual exit decisions are formed based on the assessment of the scenario from a subjective perspective. Besides, some rules are developed from specific application models. Wu and Fan [47] introduced a mixture of competitive and concessional strategies into the CA model to determine the individual probability to access a void cellular grid. In the traditional shortest distance strategy $[2,41]$, the 
main factor that affects the exit decision is the distance. And the evacuated individuals always choose the exit with the shortest distance. However, in the shortest time strategy [48], individual decisions are mainly influenced by congestions at exits. They prefer the exit which takes them the shortest time to escape. An optimized strategy combining the two strategies will perform better in simulating the behavioral logic of evacuating people. Besides, a crucial aspect of multiexit evacuation processes is that the decisionmaking behavior of humans in emergency escape scenarios is dynamic $[18,20]$. Evacuees crowded in confined spaces often tend to revisit and update their decisions dynamically in response to the changes in their surroundings to ensure that they take the best strategy with available information they collected. Zainuddin and Shuaib $[49,50]$ have developed a rule-based exit choice model called the ZM model. Then, they endowed the evacuees with the ability to leave their exits due to dynamic changes by modifying the ZM model of their excitement and the simulation accomplished optimal evacuation [40]. And the inclusion of decision adaption module was proved to make a substantial difference in enhancing the accuracy of simulations. Therefore, in addition to the design of scenarios and decision-making rules, the decision adaption of evacuees plays an important role in establishing a more realistic exit decision model.

\section{Dynamic Exit Decision Model}

3.1. Pedestrian Movement Module. In this work, we refer to the social force model presented by Helbing $[3,4]$ to simulate pedestrian behaviors. The model states that the pedestrians are self-driven particles controlled by two sources of social force: the desired force toward a target $\left(F_{i}\right)$, and the repulsive force from a neighbor pedestrian $\left(F_{a b}\right)$ or an obstacle $\left(F_{a w}\right)$. For pedestrian $a$, the resultant force $F_{a}$ can be expressed as follows:

$$
\begin{aligned}
F_{a} & =m_{a} \frac{\mathrm{d} v_{a}}{\mathrm{~d} t} \\
& =F_{i}+\sum_{a \neq b} F_{a b}+\sum F_{a w},
\end{aligned}
$$

where $b, w$, and $m_{a}$ correspond to the neighbor pedestrians, obstacles, and the body mass of $a$, respectively. These social forces have been formulated in equations (2)-(4) consistent with the conceptions proposed in $[3,4]$ :

$$
\begin{aligned}
F_{i}= & \frac{m_{a}}{\tau} \times\left(v_{a}^{*} e_{a}^{*}-v_{a}\right) \\
F_{a b}= & n_{a b} \times A_{a} \exp \left[\frac{r_{a b}-d_{a b}}{B_{a}}\right]+\left(n_{a b} k_{1}+n_{a b}^{\prime} k_{2} \triangle v_{a b} t_{a b}\right) \\
& \times\left(r_{a b}-d_{a b}\right) g
\end{aligned}
$$

$$
\begin{aligned}
F_{a w}= & n_{a w} \times A_{a} \exp \left[\frac{r_{a w}-d_{a w}}{B_{a}}\right]+\left(n_{a w} k_{1}-n_{a w}^{\prime} k_{2} v_{a} t_{a w}\right) \\
& \times\left(r_{a}-d_{a w}\right) g .
\end{aligned}
$$

In these equations, the quantity $v_{a}^{*}$ and the vector $e_{a}^{*}$, respectively, represent the desired velocity and direction of pedestrian $a$, while $v_{a}$ denotes the actual velocity which is generally less than $v_{a}^{*}$. Each pedestrian may either select an individual direction or follow the average direction of his neighbors. The term $\tau$ means a certain characteristic time in which a person can change his actual velocity. The constant quantities $A_{a}$ (a parameter that measures the psychosocial forces on individuals who are new to contact but not deformed), $B_{a}$ (the range of forces between pedestrians), and $k_{1}$ and $k_{2}$ (friction force parameters) are all calibration parameters. The $(r-d)$ is a specific function that returns the vector of deformation for pedestrians. The quantity $d_{a b}$ indicates the distance between the pedestrians' centers of mass, and the body radius of pedestrian $a$ is defined by $r_{a}$. The vectors $n_{a b}$ and $n_{a b}^{\prime}$ signify normal and tangent directions.

Figure 1 shows pedestrian $a$ moving toward his destination, which can often be simulated with path planning algorithms such as Dijkstra [51] and A* [37]. Pedestrian $a$ will shift to another position at the speed $v_{a}^{*}$ unless there are other people or obstacles interfere with his movement, which will lead to a lower speed $v_{a}$ even if he tends to move faster.

3.2. Exit Choice Module. The original social force model is not preferred in resolving multiexit evacuation unless there is a standard rule for self-driven particles to choose an exit from a set of exits. To overcome this deficiency, we integrate an exit selection strategy into the social force model by providing individuals with assessments of evacuation process and decisions to choose a favorite exit. In the exit choice model, the agents observe the others in front of them and react by choosing the best destination for the shortest estimated evacuation time. They are able to reckon in walking time and queuing time with the length of evacuation route and the clogging individuals at exits, and then the agents can make decisions based on their predictions. Conditions of exits are considered as well.

At each time step $\Delta t$, which is assumed for the interval time of the assessment in an evacuation process, the pedestrian $a$ may predict the future situation of his chosen exit and make a new decision to replace his current destination. This is achieved when the new choice acquired a shorter estimated evacuation time than the current choice. If the exits are numbered in a certain order, the decision function $H_{a}$ traverses all the indexes of the collection and returns the 


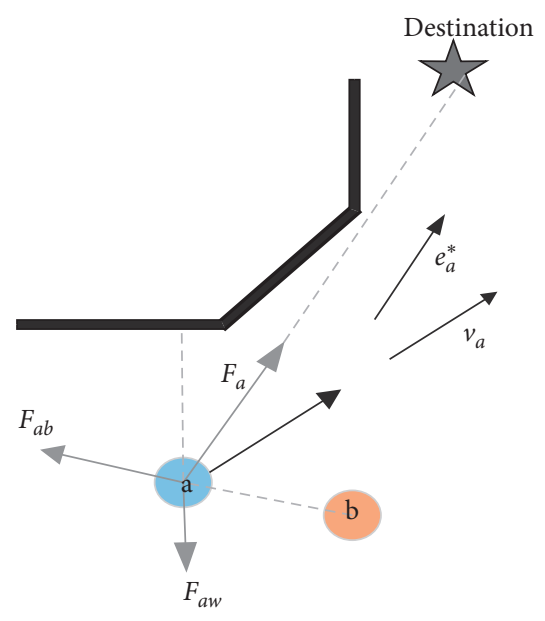

Figure 1: Social force model.

exit sequence number corresponding to the minimum estimated evacuation time. The minimum index will be accepted if there are several values that come to the same estimated time. Decision function $H_{a}$ of pedestrian $a$ is established, shown as follows:

$$
H_{a}=\min \left\{\operatorname{index}\left\{\min _{n}\left(T_{R}^{a}+\rho_{a} \times T_{Q}^{a}\right)\right\}\right\}
$$

where $n$ represents the exit $n . T_{R}^{a}$ is the minimum time of pedestrian $a$ walking freely from current position to the exit with the shortest route, and $T_{Q}^{a}$ is the estimated queuing time of pedestrian $a$ since exits are congestion bottlenecks of evacuation. They are defined by equations (6) and (7), respectively. The variable $\rho$ indicates the cognitive capability of the crowd. Accordingly, if individual $a$ is well-judged ( $\rho=1$ in particular), then the cognition of the time would be more accurate. If the rational factor is low, such as $\rho=0$, the individual would consider the $T_{R}^{a}$ as a major determinant of the best exit, which means the shortest distance strategy:

$$
\begin{aligned}
& T_{R}^{a}=\frac{L_{a j}(t)}{v_{a}^{*}}, \quad j \in\{1,2, \ldots, n\}, \\
& T_{Q}^{a}=\frac{Q_{a j}(t)}{E C_{j}}, \quad j \in\{1,2, \ldots, n\} .
\end{aligned}
$$

The quantity $v_{a}^{*}$ is the desired velocity in 3.1. Shortest distance $L_{a j}(t)$ can be obtained based on the position of pedestrian $a$ and exit $j$ [52]. Linear distance is used in this work:

$$
L_{a j}(t)=\sqrt{\left(x_{a}(t)-x_{j}(t)\right)^{2}+\left(y_{a}(t)-y_{j}(t)\right)^{2}} .
$$

The parameter $E C_{j}$ denotes the maximum evacuation capacity of the exit $j$. The value is equal to the maximum cross-sectional flow (the biggest number of individuals passing in a second). It is affected by some factors, such as width, height, thickness, and shape of the exit $[1,45]$. The power-law relationship of evacuation time and exit width has been proved while exponential fitting of evacuation time and exit thickness performs well [53]. The formula of EC is shown in equation (9), and $\omega_{j W}, \omega_{j C}, k_{W}$, and $k_{c}$ are weight coefficients while $W_{j}$ and $C_{j}$ signify the width and thickness of exit $j$, respectively. We determine $E C_{j}$ by rounding up the fitted evacuation efficiency to avoid the interference of pedestrian response time as far as possible:

$$
\begin{aligned}
E C_{j}= & \text { roundup } \\
& \left\{\left(\omega_{j W} \cdot \exp \left(-k_{W} \cdot W_{j}\right)+\omega_{j C} \cdot \exp \left(k_{C} \cdot C_{j}\right)\right)^{-1}\right\} .
\end{aligned}
$$

The variable $Q_{a j}$ reflects the number of queuing pedestrians crowded near the exit $j$. The size of the jam countarea $\varphi(t)$ varies according to the range of the count-area (Figure 2). In a room without obstacles, individuals in the semicircle (with $e_{1}$ as the center and $l_{a e}$ as the radius) are closer to the exit $e_{1}$ than pedestrian $a$. However, people tend to view individuals in the inscribed triangle as competitors because the light is proved to travel in a straight line and human eyes are more likely to realize the delta-shaped region. Hence, $\varphi(t)$ can be computed by

$$
\varphi(t)= \begin{cases}l_{a e}^{2} \cdot|\sin \theta|, & \theta \neq 0 \text { and } \theta \neq \pi, \\ \frac{1}{2} \cdot l_{a e}^{2}, & \theta=0 \text { or } \theta=\pi .\end{cases}
$$

\section{Simulations}

4.1. Experimental Setup. A single room is the smallest unit of personnel distribution in a building, and its egress is the main way for evacuation [54]. Referring to the research of Song et al. [37] (https://github.com/my-HenryS/multiagent-simulation), a Java-based evacuation system is developed to examine the validity of the dynamic exit decision model and study the effect of exit layout on the efficiency of evacuation quantitatively.

The simulation was performed for a group of evacuees in a room without obstacles, and the dimension of the room is $12 \mathrm{~m}$ in width and $12 \mathrm{~m}$ in length. The width of the exits is $0.8 \mathrm{~m}$. Everyone had a sweeping view of the room. The effect of the number of exits on evacuation time was investigated while other factors were neglected. Two settings were simulated. Scenario 1: a two-exit room $(n=2)$. Scenario 2: a three-exit room $(n=3)$. The locations of the $N$ simulated individuals were initialized randomly in the room. The values of parameters used in the simulation are estimated as in Table 1.

According to the fitting formula of the results in Song's et al. experiment [53], the values of $\omega_{j W}$ and $k_{W}$ in equation (9) are 2.805 and 0.643 , respectively. Therefore, $E C_{j}=2 \operatorname{Ped}(\mathrm{m} \cdot \mathrm{s})^{-1}$, which means that at most two people can pass through an exit every second.

Two experiments were designed with two settings, in which the exits were located on the same wall evenly and symmetrically. Experiment 1 was carried out in Scenario 1 while Experiment 2 was simulated in Scenario 2. Each experiment consisted of two parts: an experimental group for 


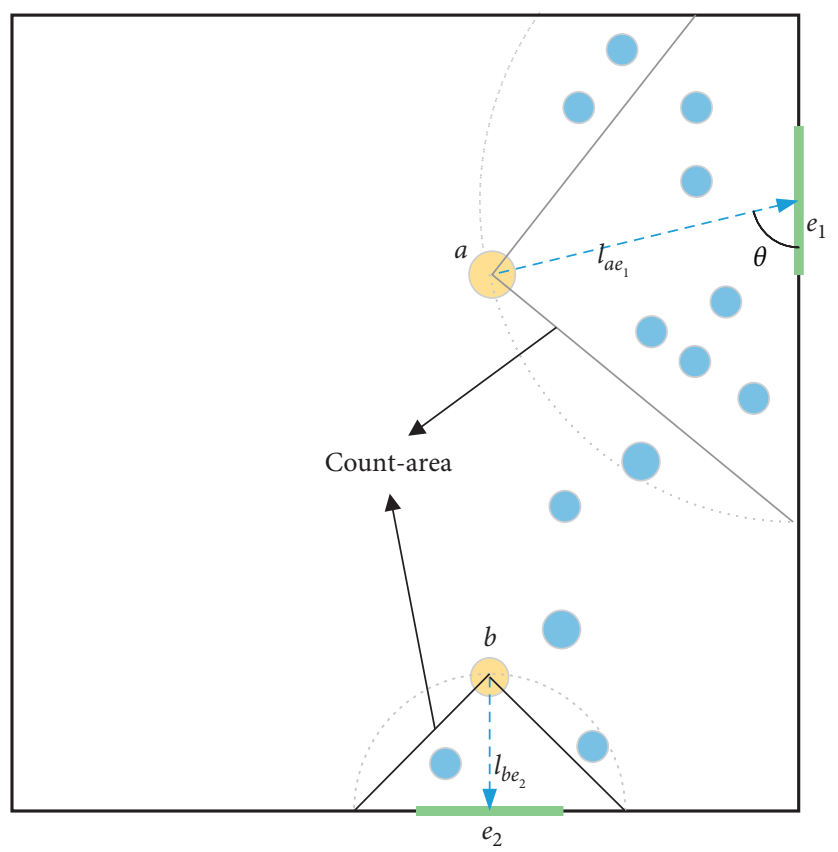

Figure 2: Schematic illustrations of the count-area.

TABle 1: Parameters used in the simulation models $[3,4,33,40,49]$.

\begin{tabular}{|c|c|}
\hline Values & Descriptions \\
\hline$m=[77-83] \mathrm{kg}$ & Pedestrians' mass \\
\hline$r=[0.25-0.30] \mathrm{m}$ & Pedestrians' radius \\
\hline$v^{*}=3 \mathrm{~m} / \mathrm{s}$ & The desired speed \\
\hline$\tau=0.5 \mathrm{~s}$ & An average reaction time of pedestrians \\
\hline$A=2000 \mathrm{~N}$ & Strength of the repulsive social force \\
\hline$B=0.8$ & Characteristic distance of the repulsive force \\
\hline$k_{1}=1.4 \times 10^{5} \mathrm{~kg} / \mathrm{sec}^{2}$ & Strength of the contact (pushing) force \\
\hline$k_{2}=2.4 \times 10^{5} \mathrm{kgm}^{-1} \mathrm{~s}^{-1}$ & Coefficient of the friction force \\
\hline$E C_{j}=2 \operatorname{Ped}(\mathrm{m} \cdot \mathrm{s})^{-1}$ & Evacuation capacity of an exit obtained by simulation \\
\hline$\Delta t=[15-30] \mathrm{s}$ & Each time step \\
\hline$\rho=[0-1]$ & The cognitive capability of pedestrians \\
\hline$N=[25-150]$ & Initial number of pedestrians \\
\hline$w=0.8 \mathrm{~m}$ & Width of exits \\
\hline
\end{tabular}

the dynamic exit decision model (EDM) and a control group for the original social force model (SFM). Table 2 shows all the experimental conditions. We conducted fifty repeated trials for each experimental condition.

In order to focus on the efficiency of two models, evacuees in SFM have the desired destinations pointing to the nearest exit, that is, the shortest distance strategy. Evacuees know the exit location all the time and try to escape on their own, and neither herding behaviors nor leaderships are allowed during the process.

4.2. The Process of Evacuation. The procedure of evacuation can be split into three stages as shown in the snapshot (Figure 3) from the experimental process of EDM. The first stage: the requested number of pedestrian particles with different radii is generated at random positions in the simulation scenario and they start their movement towards the exit they chose at the same time. The second stage: part of the simulated pedestrians choosing different exits have conflicts and some of them change their exit choices. As time goes by, people increase at each exit and the wandering phenomenon of pedestrians [43] appears. The last stage: the weave and conflict of particles gradually disappear, namely, results of exit choice are no longer changed. All evacuees separated completely and walk away through the corresponding exit, where two smooth flows occurred. There is a decrease in the crowd density near exits before the last survivor out, and then the evacuation comes to an end.

Collision is a familiar phenomenon (Figures 3(a) and $3(b))$, especially at the end of the queue where the crowd wanders as exit decisions change. This phenomenon also occurs near the exits, as individuals tend to compete when the evacuation capacity of exits is limited. And there is a selforganization phenomenon [55] in pedestrian crowds displayed in Figure 3(b), evacuees spontaneously gathered in 
TABLE 2: Characteristic conditions of experiments.

\begin{tabular}{lccc}
\hline Experiment & Scenario & Group & Initial number of pedestrians \\
\hline \multirow{2}{*}{ Experiment 1} & 1 & Experimental group (EDM) & $N=25,50,75,100,125,150$ \\
& $(n=2)$ & Control group (SFM) & (Crowd density $=0.1,0.3,0.5,0.7,0.9,1.0)$ \\
\hline \multirow{2}{*}{ Experiment 2} & 2 & Experimental group (EDM) & $N=25,50,75,100,125,150$ \\
& $(n=3)$ & Control group (SFM) & (Crowd density $=0.1,0.3,0.5,0.7,0.9,1.0)$ \\
\hline
\end{tabular}

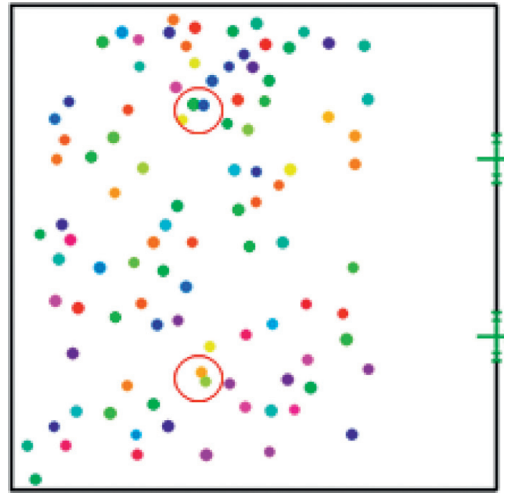

(a)

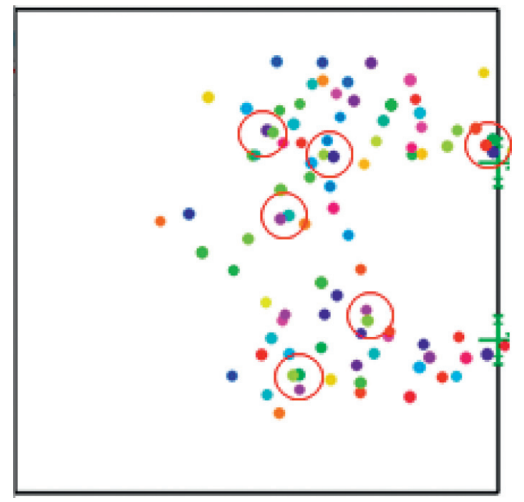

(b)

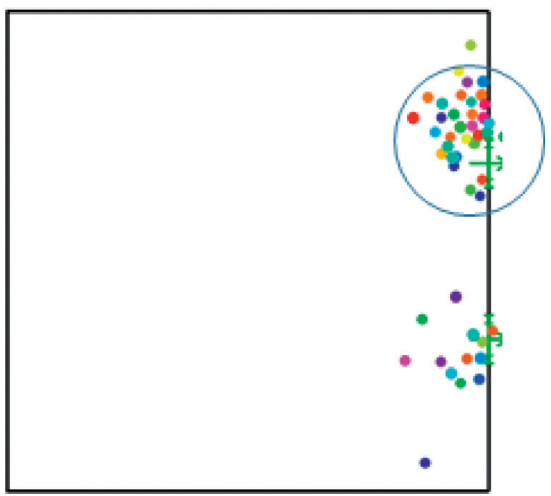

(c)

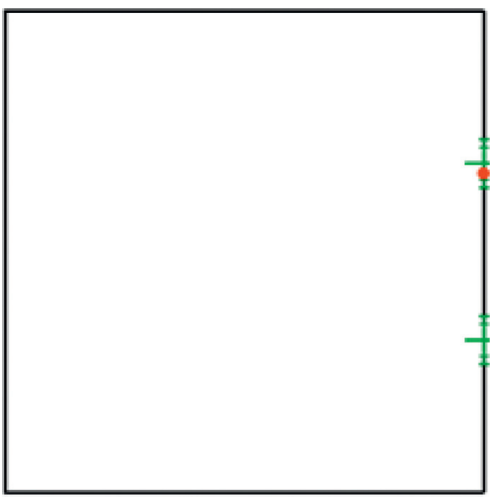

(d)

Figure 3: Snapshots of EDM (Experiment 1, $N=100$ ): (a) $t=0 \mathrm{~s}$; (b) $t=10 \mathrm{~s}$; (c) $t=35 \mathrm{~s}$; (d) $t=40.15 \mathrm{~s}$.

two streams through the exit. With the accumulation of individuals at the exits, clogging arises. An arch, which squeezed into sector shape, is formed in front of an exit, as shown in the blue circle in Figure 3(c). People who try to move faster can cause a smaller average speed, which is called the "faster-is-slower effect." The features described above are in accord with the known phenomena of evacuation.

4.3. Results and Discussion. Bad jam and inefficient use of exits caused by the consistent decision-makers of SFM differ from facts. The performance difference between EDM and SFM regarding the decision-change module was active or is not shown in Figure 4. The comparison of Figures 4(a)-4(d) indicates that the difference in the utilization of exits between EDM and SFM is obvious. Available exits are not efficiently used in SFM while EDM shows a balancing effect of people distribution. And the rational decision-making group of EDM, who can change their minds to a better exit, leads to a smaller and lighter crowded area exhibited in Figures $4(\mathrm{c})$ and $4(\mathrm{~d})$.

The distribution of evacuation times associated with each experimental condition is depicted in Figure 5. The plots visualize the evacuation time for the trials as well as minimum, first quartile, average, third quartile, and maximum of the observed evacuation time for each imitated scenario in simulation over 50 repeats. The blue boxes on the left of the two plots are associated with SFM while the green boxes on the right correspond to EDM. The bisector line $y=x$ is superimposed to facilitate the comparison, and the scatter of the boxes around the line is a measure of the dissimilarity between the EDM and the SFM outputs.

Almost all averages of EDM line up under the bisector line while the values of SFM are near the line or even scatter in the upper half of the figures. The variation in evacuation time of different initial pedestrian quantities is similar when the intelligent behaviors of people are considered. A larger 


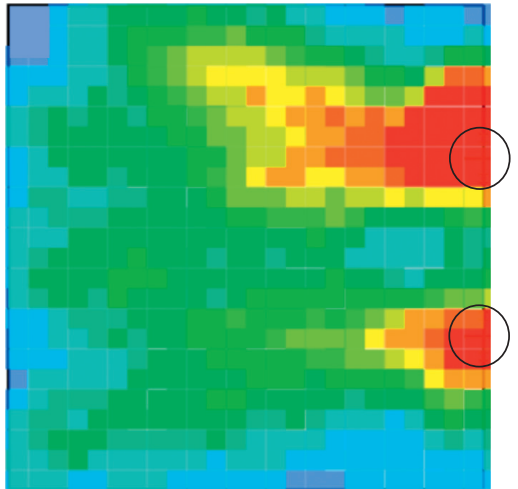

(a)

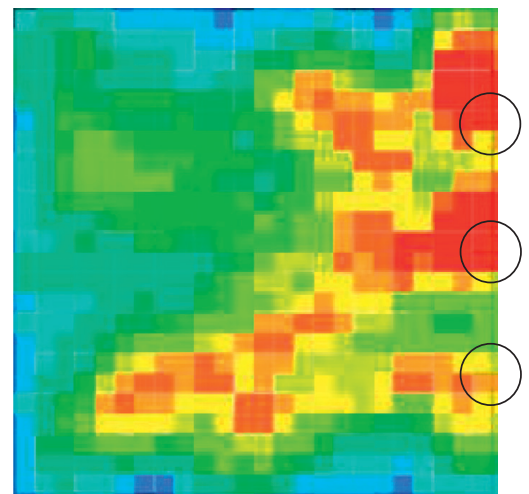

(b)

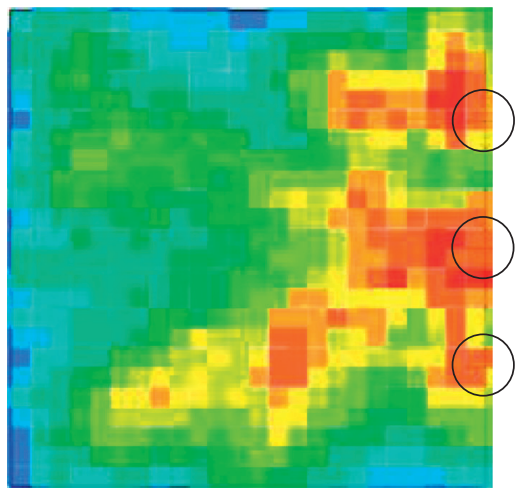

(d)

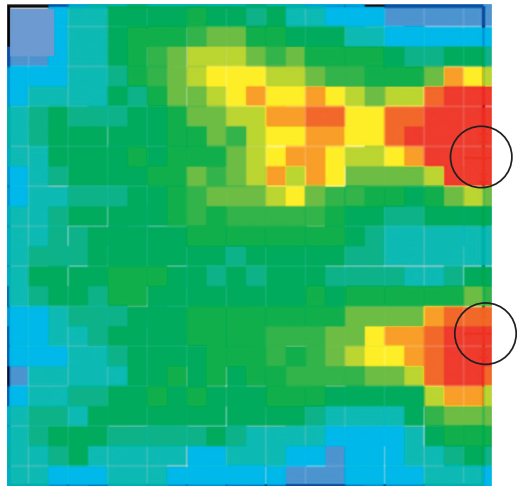

(c)

FIgURE 4: Density map of two models $(N=100)$ (the positions of exits are circled): (a) Experiment 1 of SFM; (b) Experiment 2 of SFM; (c) Experiment 1 of EDM; (d) Experiment 2 of EDM.

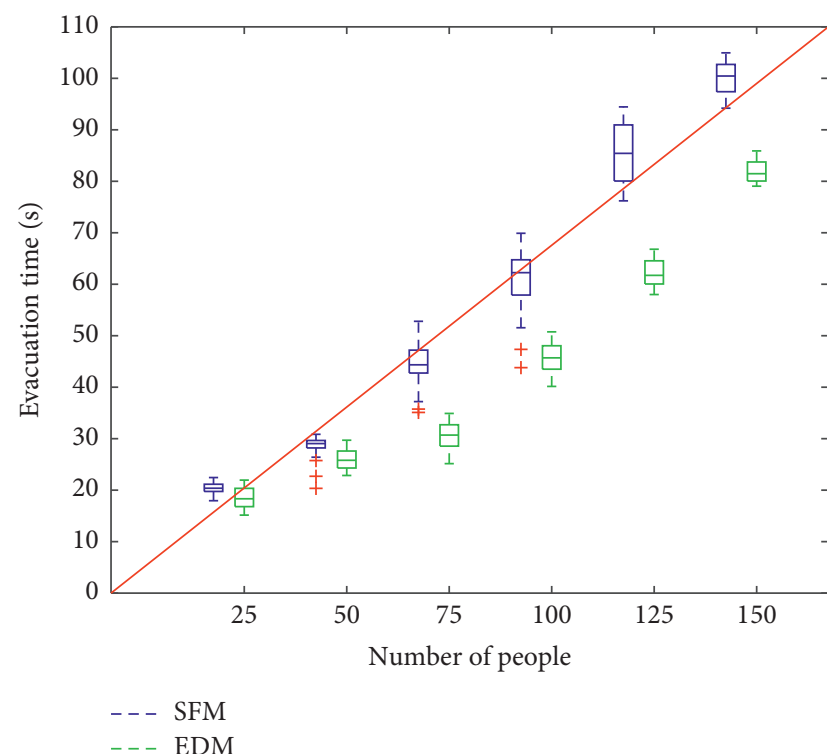

(a)

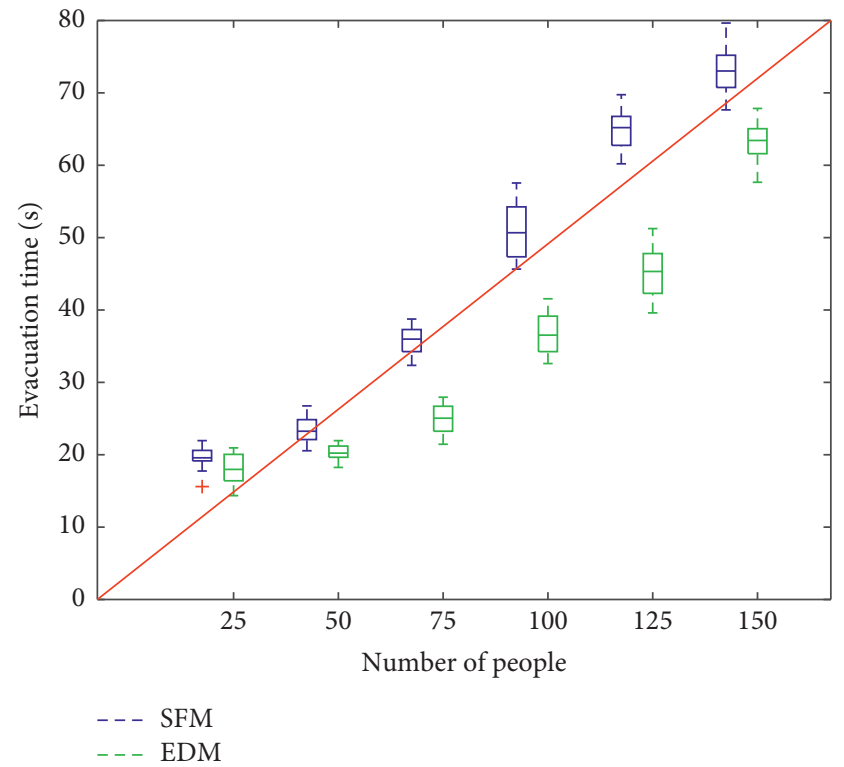

(b)

FIgURE 5: Box plots for multiexit evacuation time calculated 50 times of simulation repetitions, corresponding with the setups in Table 2: (a) Experiment 1: $n=2$; (b) Experiment 2: $n=3$. 
scatter of evacuation times is measured when setups of a dense crowd are compared to those of sparse people.

As observed, both plots reveal a degree of pattern resemblance between the obtained outcomes from the two-exit experiment and the three-exit experiment. They both indicate that the social force model performs badly in terms of evacuation time compared with the exit decision model. The model (EDM) which takes pedestrians' choices into consideration is more efficient and realistic than the pure mechanical model (SFM) when there is a large crowd density. However, the extent of the difference in evacuation time associated with two models appears to be smaller when the number of individuals is less than fifty. In general, EDM is almost 20\% (average of the ratios of the increased efficiency in each case) more efficient than SFM, as shown in Table 3.

The sensitivity analysis studies the changing of evacuation time with crowd density in a two-exit simulation scenario (Figure 6), based on $\rho=0.1,0.3,0.5,0.7,0.9,1.0$. It is found that evacuation time will increase with the crowd density rising and the slope of the curves comes to maximum when $\rho=0.1$. The evacuation time has significant differences in the condition of different system scales, and big gatherings can lead to longer evacuation. In the case of fixed pedestrian density, there is a decreasing trend in the process of pedestrian evacuation time changing with individual cognitive capability. The more rational people are, the more accurate the judgment of queuing time is, and evacuees are more likely to make wise choices to achieve a higher evacuation efficiency.

Figure 4(d) displays that local congestion also occurs far away from exits when multiple exits are on the same side of a room, which can be alleviated through exits on different sides, as shown in Figure 7. The alteration of target exit changes the direction of pedestrian movement and reduces congestion areas. However, collisions enhance the resistance of movement which may increase evacuation time. During the experiments, locations of exits result in distributions of pedestrian concentration areas. The conflicts are particularly intense when two exits are too close to each other. It is shown that the exit location is a key factor in pedestrian evacuation.

\section{Optimization of Two-Exit Layout}

Dual exits are the most common exit configurations. This section focuses on the question that how positional features of egress affect collective outcomes in a room with two exits. An evacuation system is simulated in three typical architectural layouts (Figure 8), where the width of egress is $0.8 \mathrm{~m}$ and the number of pedestrians is set to $N=150$. Experimental data for each configuration are obtained by averaging 1125 independent simulation runs.

The center points of exit $e_{1}$ and $e_{2}$ are $\left(x_{1}, y_{1}\right)$ and $\left(x_{2}, y_{2}\right)$, respectively, in the coordinate system $\left(x_{1}, x_{2}, y_{1}, y_{2} \in[0.4,11.6]\right)$. From Figure 8 , it is noted that $x_{1}=x_{2}=12$ when two exits on the same side, $x_{1}=12$ and $y_{2}=0$ for the adjacent exits and $x_{1}=12$ and $x_{2}=0$ for the parallel exits.
5.1. Two Exits on the Same Wall. Figure 9(a) illustrates how the evacuation time performs as the coordinates of two exits changing on the same wall. The evacuation time is shorter when two exits are located anywhere on the wall instead of corners. In addition, the escape time of unilateral configuration approximately symmetric to the line $y_{1}=y_{2}$, which means the evacuation efficiency will not be affected by the exchange of two identical exits. When the coordinates of two exits coincide $y_{1}=y_{2}$, the evacuation time reaches its maximum as there is only one exit with a width of $0.8 \mathrm{~m}$.

As shown in Figure 10, the evacuation time descends firstly and then ascends with the increase of the separation of two exits. The evacuation efficiency generally reaches the maximum when at least one of two exits is near the center of the wall $(y=6)$. If one exit is near the corner, the best position for the other exit to achieve a better evacuation is near the center. It is not conducive to evacuation whether the separation of two exits is too large or too small. The situation that one exit is far away from the other will lead to a long average distance to escape, resulting in longer time intervals between the former and the latter who reach the exit. The time intervals of individuals' escape with long paths relieve the pressure near exits, where pedestrian jam occurs frequently. And the local crowd density is relatively low, and thus, those pedestrians approaching the exits could proceed almost unimpeded. Although those who lag behind are inclined to run fast towards exits, they are less likely to change their destinations as they paid enough time to the current positions, and changes can lead to longer walking time.

The interference between two exits gradually enhances with $\left|y_{1}-y_{2}\right|$ reduces, in which case, pedestrians who select one exit will be hindered by others who direct their fleeing toward the other exit. More time will be spent to make decisions, especially for evacuees in the region between two exits. Individuals left in the room gather at exits and compete for rushing out, but the limitation of exits' capacity results in long waiting times and low efficiency. There is an abrupt decrease in the temporal pedestrian flow rate after a gradual increase, and the flow rate will fluctuate around a specific value, meaning a typical "faster-is-slower effect" happens. This phenomenon can also be found in works done by Wang et al. [45], Garcimartín et al. [56], and Liao et al. [57].

5.2. Two Exits on the Adjacent Wall. The relations between positions of two exits and the total evacuation time are presented in Figure 9(b). The figure is symmetrical to the line of $y_{1}+x_{2}=12$. Compared with Figure 9(a), the difference in the occupant evacuation time is minor when two exits are on the adjacent walls. The escape time fluctuates from $85 \mathrm{~s}$ to $90 \mathrm{~s}$ and rises sharply when two exits are close to the same corner.

The initial locations of evacuees were found from four regions in the simulation. The phase diagram is divided into four regions (Figure 11(a)) when two exits locate on adjacent walls. The number of individuals who leave from exit $e_{1}$ is large in region $\mathrm{A}$ and $\mathrm{C}$, but in region $\mathrm{B}$ and $\mathrm{D}$, most pedestrians tend to escape from exit $e_{2}$. This may change when the two exits are far enough apart that people in region $\mathrm{B}$ choose exit $e_{1}$ while the destination of evacuees in region $\mathrm{C}$ is 
TABLE 3: Statistical analysis of experimental results.

\begin{tabular}{|c|c|c|c|c|c|c|}
\hline \multirow{2}{*}{$\begin{array}{l}\text { Number of } \\
\text { people }\end{array}$} & \multicolumn{3}{|c|}{ Experiment $1: n=2$ (two exits) } & \multicolumn{3}{|c|}{ Experiment 2: $n=3$ (three exits) } \\
\hline & $\begin{array}{c}\text { Times of SFM } \\
\text { (s) }\end{array}$ & $\begin{array}{c}\text { Times of EDM } \\
\text { (s) }\end{array}$ & $\begin{array}{c}\text { Ratios of improvement } \\
(\%)\end{array}$ & $\begin{array}{c}\text { Times of SFM } \\
\text { (s) }\end{array}$ & $\begin{array}{c}\text { Times of EDM } \\
\text { (s) }\end{array}$ & $\begin{array}{c}\text { Ratios of improvement } \\
(\%)\end{array}$ \\
\hline 25 & 20.391 & 18.536 & 9.097 & 19.801 & 18.030 & 8.944 \\
\hline 50 & 28.627 & 25.974 & 9.267 & 23.437 & 20.260 & 13.555 \\
\hline 75 & 44.471 & 30.426 & 31.582 & 35.680 & 24.871 & 30.294 \\
\hline 100 & 60.931 & 45.660 & 25.063 & 50.979 & 36.789 & 27.835 \\
\hline 125 & 85.456 & 62.254 & 27.151 & 64.869 & 45.384 & 30.037 \\
\hline 150 & 100.189 & 81.935 & 18.220 & 73.001 & 63.102 & 13.560 \\
\hline Average & & & 20.063 & & & 20.704 \\
\hline
\end{tabular}

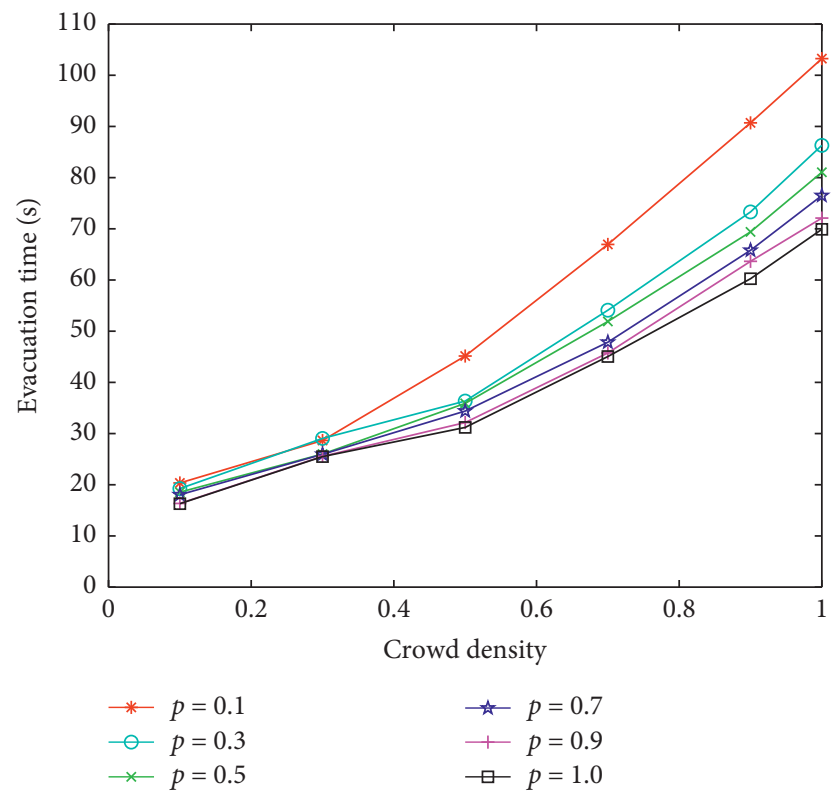

FIgURE 6: Curves of the mean evacuation time against initial density.

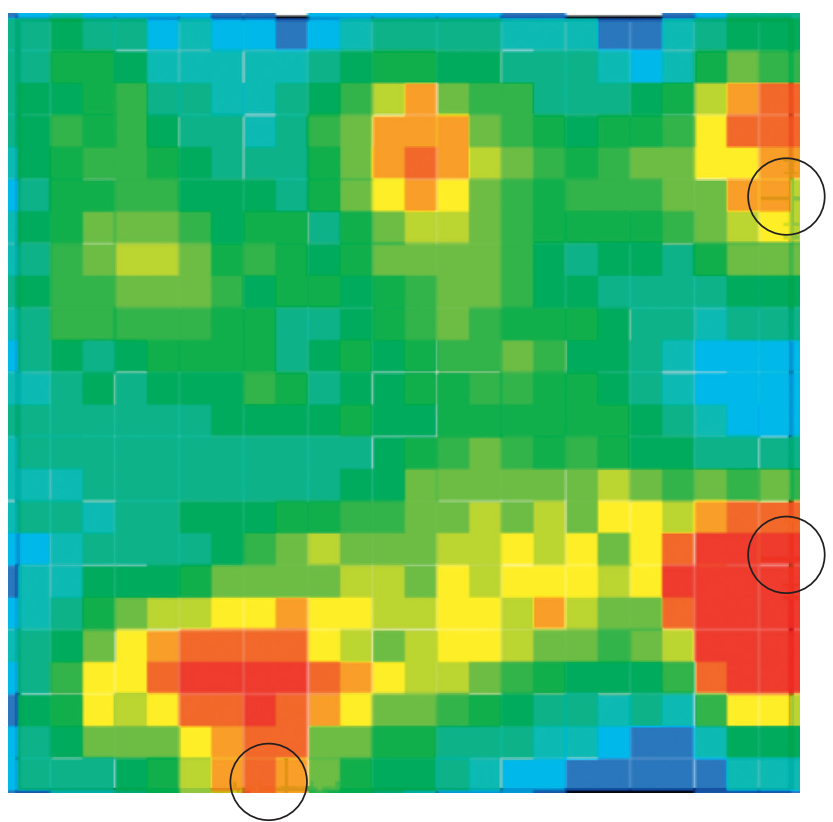

FIgURe 7: Density map of the evacuation of exits on different sides of a room (the locations of exits are circled). 


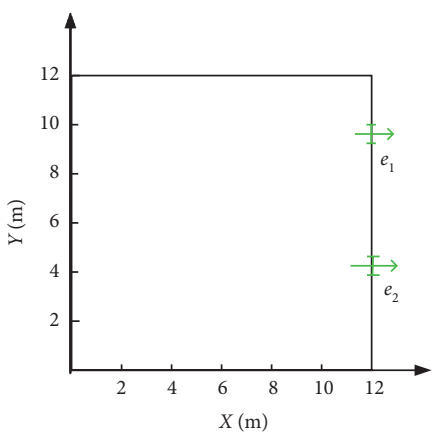

(a)

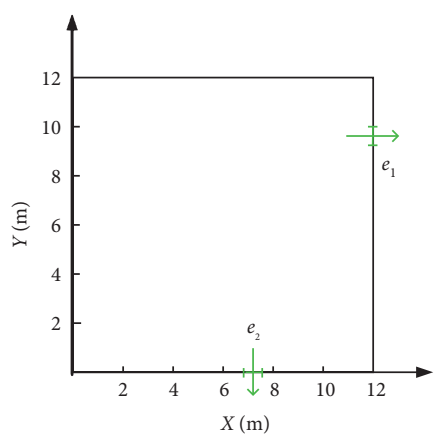

(b)

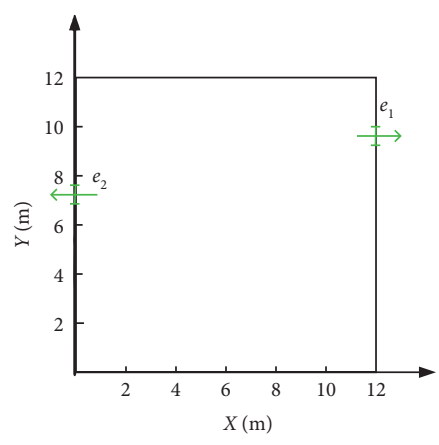

(c)

Figure 8: The configuration of a two-exit room: (a) two exits on the same wall; (b) two exits on the adjacent walls; (c) two exits on the parallel walls.

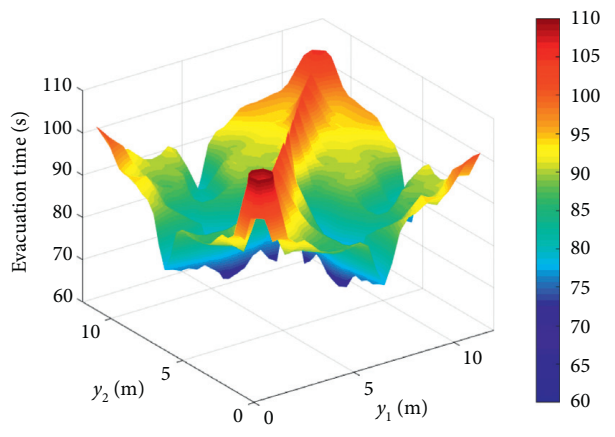

(a)

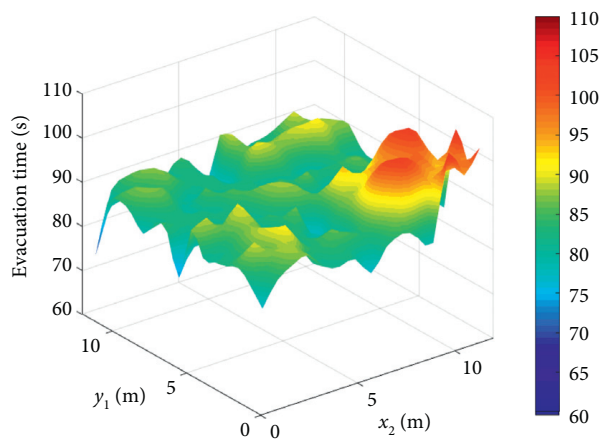

(b)

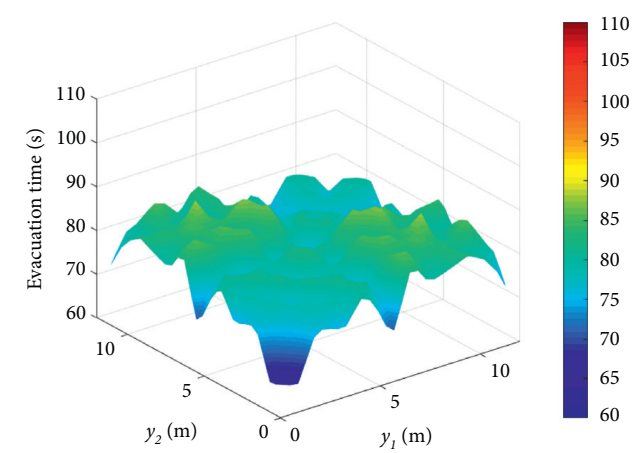

(c)

FIGURE 9: Effects of different configurations on evacuation: (a) evacuation time against $y_{1}$ and $y_{2}$ for $x_{1}=x_{2}=12$; (b) evacuation time against $y_{1}$ and $x_{2}$ for $x_{1}=12$ and $y_{2}=0$; (c) evacuation time against $y_{1}$ and $y_{2}$ for $x_{1}=12$ and $x_{2}=0$.

exit $e_{2}$. The total evacuation time is determined by the longer time of two exits. This phenomenon was confirmed in 2015. Huan-Huan et al. [58] found that the four regions have equal size under certain circumstances and the average distance of evacuees to two exits is the longest when the two exits locate near the same corner. There is a risk of pedestrian collisions around the boundary of the four regions except for two exits, because that is where the flows meet. Individuals may change their destinations here due to interference from others.

The outcomes of the comparison between the simulated measurements are summarized in Figure 11(b). The fluctuation curve of evacuation time is irregular in most cases. For the case of $y_{1}=11.6 \mathrm{~m}$ and $x_{2}=0.4 \mathrm{~m}$, two exits are located in different corners, and the utilization rate of both exits is high. The total escape time reaches a minimum of $73.66 \mathrm{~s}$. And a certain distance between exits or between the exits with a same corner is conducive to reducing the total evacuation time, although the total escape time is affected by walking time. The utilization of exits drops when they are too close to each other, because the presence of the side walls will have a negative effect on individual decisionmaking and pedestrians sway between different exits which 


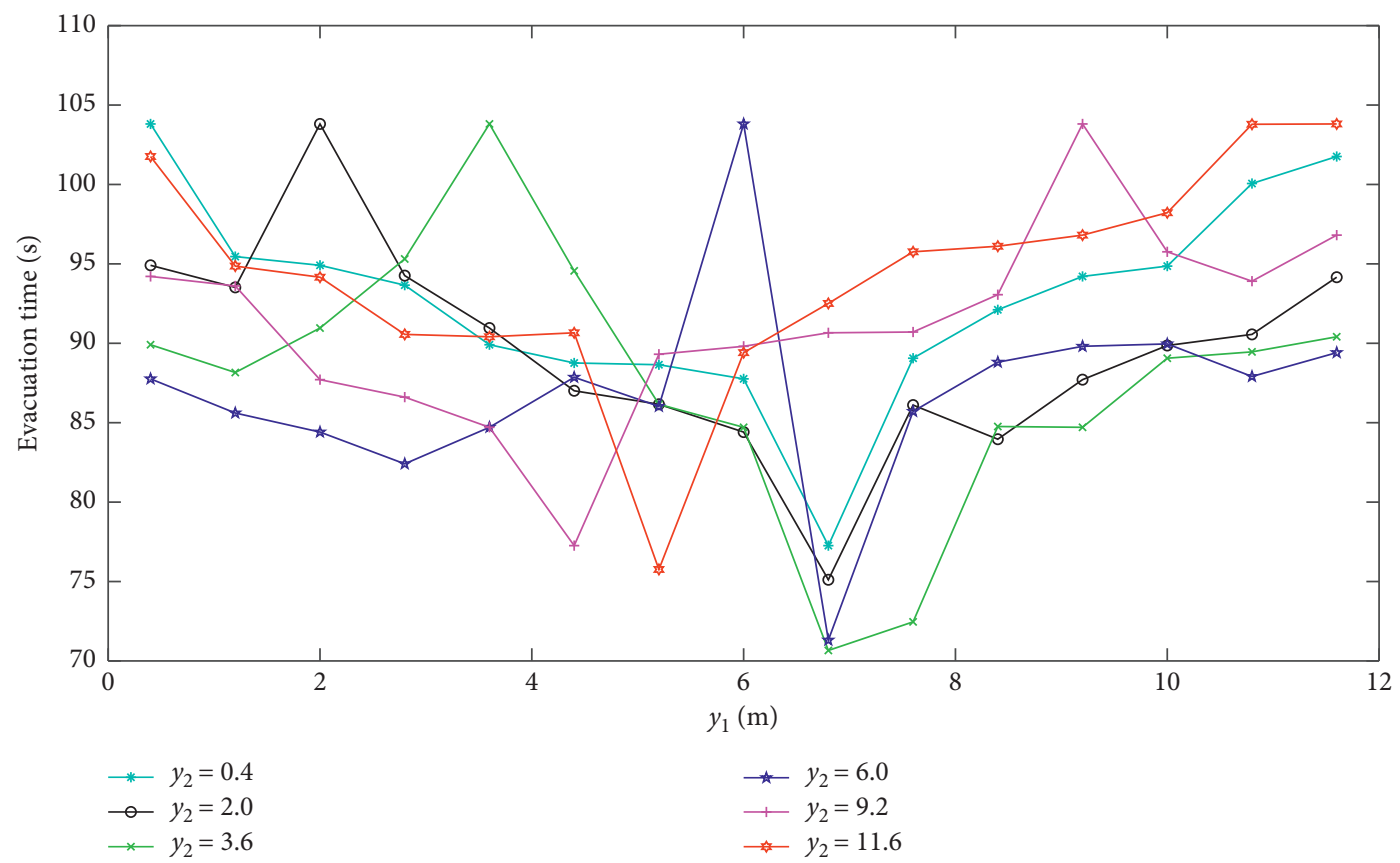

Figure 10: The relations between evacuation time and $y_{1}$ for $y_{2}=0.4 \mathrm{~m}, 2.0 \mathrm{~m}, 3.6 \mathrm{~m}, 6.0 \mathrm{~m}, 9.2 \mathrm{~m}, 11.6 \mathrm{~m}$ when two exits on the same wall.

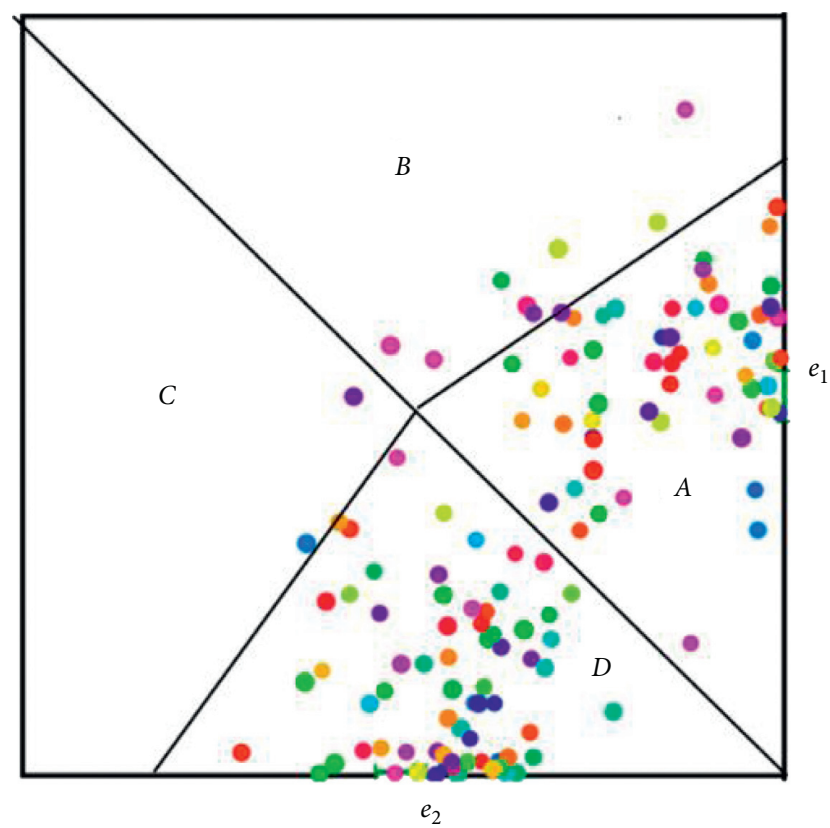

(a)

FIgURE 11: Continued. 


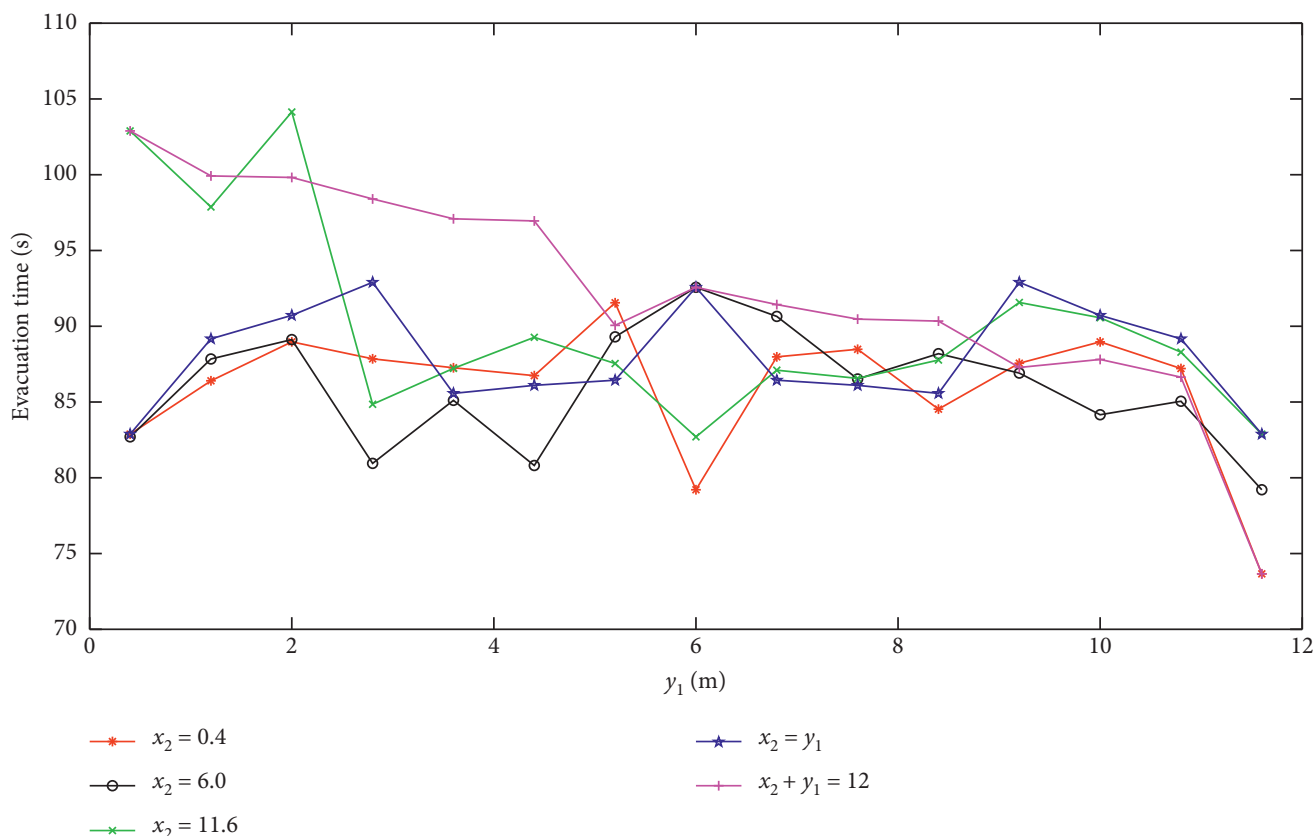

(b)

Figure 11: The relations between evacuation time and exit positions when two exits on the adjacent walls: (a) phase diagram for symmetrical adjacent exits; (b) evacuation time against $y_{1}$ for $x_{2}=0.4 \mathrm{~m}, 6 \mathrm{~m}, 11.6 \mathrm{~m}, y_{1}$ and $y_{1}+x_{2}=12$.

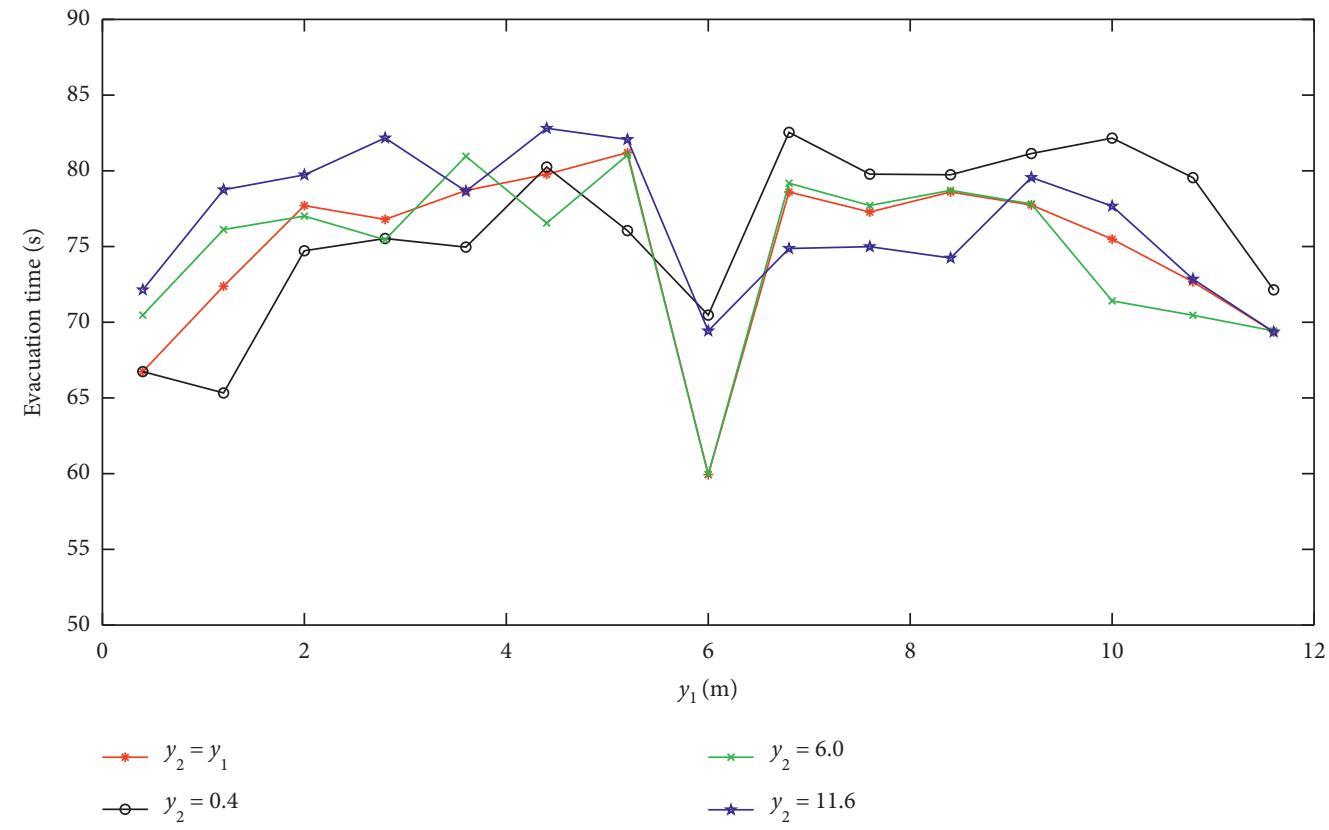

Figure 12: The relations between evacuation time and $y_{1}$ for $y_{2}=y_{1}, 0.4 \mathrm{~m}, 2.0 \mathrm{~m}, 3.6 \mathrm{~m}$ when two exits on the parallel wall.

move towards a stalemate. In the simulation, the crowd density of two exits will reach a certain equilibrium state with the flow of people moving, and the queuing time is limited by the exit capacity.

5.3. Two Exits on the Parallel Walls. Figure 9(c) displays that evacuation time fluctuates with the position changes of two exits, and the diagram is found to be symmetrical with respect to the center point $(6,6)$. The average evacuation time under the parallel scheme is the shortest among all the schemes, and the maximum is less than $90 \mathrm{~s}$. The exits locate near the corners optimize the evacuation effect to some extent, in which case, pedestrians have to move a long average distance to reach their target exit. Once a pedestrian 
makes his choice, he is reluctant to spend more estimated walking time to change the decision. They can pass through the exits quickly as there is no serious congestion in front of exits. And the result shows that the distribution of the number of evacuees chosen different exits in the evacuation is nonuniform, which can be influenced by the initial locations of people. For the situation of one exit locates around the corner $\left(y_{2}=0.4 \mathrm{~m}\right.$, for example), the evacuation time increases with the reduction of the coordinate difference between exit $e_{1}$ and the center point $(12,6)$. However, the escape time declines when $y_{1}=6 \mathrm{~m}$.

In the case of opposite exits, the evacuation time is shorter than other configurations. We noticed that the phenomenon of crowd wandering caused by decision changes is quite frequent, especially in the rectangular area of two exits. And the crowd size at each exit is similar. The average queuing time to exit is the main factor while the difference in average walking distance of pedestrians is small. A lot of pedestrians tend to be attracted by the uncrowded exit, and they spend more time queuing and detour frequently.

Figure 12 illustrates four curves which have the same trend that the evacuation time fluctuates with increasing the value of $y_{1}$. It shows that either two exits locate in the center of two walls or near the corner are wise strategies when setting the exits' positions for evacuation. In particular, the former setup corresponds to the minimum evacuation time of $59.94 \mathrm{~s}$.

\section{Conclusions}

In this paper, a dynamic exit decision model (EDM) is proposed to simulate decisions of evacuees in the multiexit evacuation, and three different exit layouts are outlined and quantitatively compared through simulations of the model in a regular room without obstacles. An exit selection strategy that relies on the shortest estimated time is introduced to solve the inapplicability of the original social force model. The simulated individuals will choose the best suitable exits by comparing the total estimated time of walking and queuing. The cognitive capability $\rho$ of individuals plays an important role in the decisions as well. We compared the effects of traditional shortest distance evacuation strategies and the optimized evacuation strategies. Two experiments were identified in two scenarios, in which EDM was the experimental group and SFM was the control group. The experiments systematically tested the effect of evacuees' choices on the effectiveness of evacuation, and it is confirmed that EDM is almost $20 \%$ more efficient than SFM. We also showed how the simulation outcomes of the crowd evacuation through two exits can be significantly sensitive to the specification of the cognitive capability $\rho$. When $\rho=0$, the exit selection strategy is equivalent to the shortest distance strategy. The pedestrian jam occurs and gets worse with initial crowd density rising since the width of exits is fixed.

Various exit layouts were analyzed to evaluate design optimization as it relates to the effects of evacuation. Different layouts result in different times for evacuees escaping, and preliminary results indicate that evacuation time will be improved with the changes of exit locations. There are three layouts investigated: two exits located on the same wall, on the adjacent walls, and on the parallel walls. Our simulation analyzes produced three fluctuating graphs of total evacuation time versus locations of two exits. The total escape time ranges from $70 \mathrm{~s}$ to $105 \mathrm{~s}$ when two exits locate on the same side or adjacent walls, while the time of parallel exits fluctuates from $59 \mathrm{~s}$ to $90 \mathrm{~s}$. It seems that two exits outperform one exit and two parallel exits are a wise choice for urgent evacuation. In addition, we summarized three findings as follows. First, it is not conducive to evacuation whether the separation of two exits is too large or too small. A long distance between two exits has a good impact on alleviating congestion and most pedestrians proceed almost unimpeded through two exits. However, it results in a long average walking time for evacuees. Although a short distance leads to a short average walking time, the phenomenon of individuals' wander occurs frequently as evacuees have difficulties in making decisions with a lot of distractions (other people [19] or the side walls [18]) if two exits are close to each other or to the corners. The efficiency decreases with serious pedestrian jams. Second, two symmetric exits on the same wall, two exits locate near different corners on the adjacent walls and two opposite exits locate near the corner or in the middle of the parallel walls performed well during urgent evacuations under their respective conditions. Finally, in some particular configurations, an even symmetry is found more efficient than an asymmetric distribution of exits, which is consistent with the results in $[59,60]$.

The observations above have important implications for evacuation planning. First, we propose an approach for improving crowd modeling, which provides new insight into the pedestrian decision-making process. Secondly, we confirmed that the symmetrical design principle is indeed conducive to evacuation. Furthermore, our findings from simulated scenarios may help formalize the quantification of exit locations by abstracting the concrete evacuation scenarios into single grids to help managers to design solutions that can facilitate evacuations in all kinds of evacuations. Although our work contributes to an understanding of emergency evacuation, several questions need to be addressed in future research. First, note that the social force model cannot perfectly simulate the movement of pedestrians during emergency evacuations. Some problems, such as unrealistic speed changes and collisions to wall [37], still require further exploration. Second, the analyses reported in this work do not consider "cooperative behaviors" [22], "herding behavior" [5], "leadership" [61], and "physical aggressiveness" [36], which might be possibilities of affecting choices of evacuees in emergencies. Third, the inaccurate computation of exit capacity (EC), which simply superimposes the influence of the width and thickness, might lead to wrong instructions and imprecise evacuation results. Finally, the experiments were calibrated in simulated scenarios without obstacles and dynamic changes. And it is of vital importance to consider other objective factors during evacuation simulations. In addition, complex application scenarios were not considered in this research to meet a 
variety of abstract analysis needs in normal evacuations. Therefore, it is a challenge to find a suitable measurement standard to summarize more general rules from a more specific data level. We can only use some qualitative descriptions to explain our findings. Future research drawing from a more practical evacuation is needed to address questions of evacuation simulation.

\section{Data Availability}

The data used to support the findings of this study are available from the corresponding author upon request.

\section{Conflicts of Interest}

The authors declare that they have no conflicts of interest.

\section{Acknowledgments}

This work was supported by a grant from the National Natural Science Foundation of China (project no. 71671128).

\section{References}

[1] A. Johnson, S. Zheng, A. Nakano, G. Schierle, and J. H. Choi, "Adaptive kinetic architecture and collective behavior: a dynamic analysis for emergency evacuation," Buildings, vol. 9, no. 2, 2019.

[2] H. A. Kurdi, S. Al-Megren, R. Althunyan, and A. Almulifi, "Effect of exit placement on evacuation plans," European Journal of Operational Research, vol. 269, no. 2, pp. 749-759, 2018.

[3] D. Helbing and P. Molnár, "Social force model for pedestrian dynamics," Physical Review E, vol. 51, no. 5, pp. 4282-4286, 1995.

[4] D. Helbing, I. Farkas, and T. Vicsek, "Simulating dynamical features of escape panic," Nature, vol. 407, no. 6803, pp. 487-490, 2000.

[5] D. J. Low, "Following the crowd," Nature, vol. 407, no. 6803, pp. 465-466, 2000.

[6] D. Helbing, I. J. Farkas, and T. Vicsek, "Freezing by heating in a driven mesoscopic system," Physical Review Letters, vol. 84, no. 6, pp. 1240-1243, 2000.

[7] W. Lei, A. Li, R. Gao, N. Zhou, S. Mei, and Z. Tian, "Experimental study and numerical simulation of evacuation from a dormitory," Physica A: Statistical Mechanics and Its Applications, vol. 391, no. 21, pp. 5189-5196, 2012.

[8] X. Shi, Z. Ye, N. Shiwakoti, D. Tang, C. Wang, and W. Wang, "Empirical investigation on safety constraints of merging pedestrian crowd through macroscopic and microscopic analysis," Accident Analysis \& Prevention, vol. 95, pp. 405416, 2016.

[9] M. Moussaid, D. Helbing, and G. Theraulaz, "How simple rules determine pedestrian behavior and crowd disasters," Proceedings of the National Academy of Sciences, vol. 108, no. 17, pp. 6884-6888, 2011.

[10] S. Gwynne, E. R. Galea, M. Owen, P. J. Lawrence, and L. Filippidis, "A review of the methodologies used in the computer simulation of evacuation from the built environment," Building And Environment, vol. 34, no. 6, pp. 741-749, 1999.

[11] A. Kneidl, D. Hartmann, and A. Borrmann, "A hybrid multiscale approach for simulation of pedestrian dynamics,"
Transportation Research Part C: Emerging Technologies, vol. 37, pp. 223-237, 2013.

[12] R. M. Tavares, "Design for horizontal escape in buildings: the use of the relative distance between exits as an alternative approach to the maximum travel distance," Safety Science, vol. 48, no. 10, pp. 1242-1247, 2010.

[13] H. Gao, B. Medjdoub, H. Luo, H. Zhong, B. Zhong, and D. Sheng, "Building evacuation time optimization using constraint-based design approach," Sustainable Cities And Society, vol. 52, 2020.

[14] H. Vermuyten, J. Beliën, L. De Boeck, G. Reniers, and T. Wauters, "A review of optimisation models for pedestrian evacuation and design problems," Safety Science, vol. 87, pp. 167-178, 2016.

[15] M. Haghani, "Optimising crowd evacuations: mathematical, architectural and behavioural approaches," Safety Science, vol. 128, 2020.

[16] G. Albi, M. Bongini, E. Cristiani, and D. Kalise, "Invisible control of self-organizing agents leaving unknown environments," Siam Journal on Applied Mathematics, vol. 76, no. 4, pp. 1683-1710, 2016.

[17] M. Davidich and G. Köster, "Towards automatic and robust adjustment of human behavioral parameters in a pedestrian stream model to measured data," Safety Science, vol. 50, no. 5, pp. 1253-1260, 2012.

[18] M. E. Yuksel, "Agent-based evacuation modeling with multiple exits using neuroevolution of augmenting topologies," Advanced Engineering Informatics, vol. 35, pp. 30-55, 2018.

[19] M. Kinateder, B. Comunale, and W. H. Warren, "Exit choice in an emergency evacuation scenario is influenced by exit familiarity and neighbor behavior," Safety Science, vol. 106, pp. 170-175, 2018.

[20] Y. Li, H. Jia, J. Li, J. Gong, and K. Sun, "Pedestrian evacuation behavior analysis and simulation in multi-exits case," International Journal of Modern Physics C, vol. 28, no. 10, 2017.

[21] M. Moussaid, N. Perozo, S. Garnier, D. Helbing, and G. Theraulaz, "The walking behaviour of pedestrian social groups and its impact on crowd dynamics," PLoS One, vol. 5, no. 3, p. 7, 2010

[22] Y. Cheng and X. Zheng, "Can cooperative behaviors promote evacuation efficiency?” Physica A: Statistical Mechanics and Its Applications, vol. 492, pp. 2069-2078, 2018.

[23] C. Arteaga and J. Park, "Building design and its effect on evacuation efficiency and casualty levels during an indoor active shooter incident," Safety Science, vol. 127, 2020.

[24] N. Khamis, H. Selamat, F. S. Ismail, O. FarouqLutfy, M. FadzliHaniff, and I. N. A. MohdNordin, "Optimized exit door locations for a safer emergency evacuation using crowd evacuation model and artificial bee colony optimization," Chaos Solitons \& Fractals, vol. 131, p. 109505, 2020.

[25] H. C. Braga, G. F. Moita, and P. E. M. D. Almeida, "The influence of the location of emergency exits over the distance to be covered to the exit of an environment," Ambiente Construído, vol. 19, no. 2, pp. 219-232, 2019.

[26] Q.-F. Gao, Y.-Z. Tao, Y.-F. Wei, C. Wu, and L.-Y. Dong, "Simulation-based optimization of inner layout of a theater considering the effect of pedestrians," Chinese Physics B, vol. 29, no. 3, 2020 .

[27] X. Shi, Z. Ye, N. Shiwakoti, D. Tang, and J. Lin, "Examining effect of architectural adjustment on pedestrian crowd flow at bottleneck," Physica A: Statistical Mechanics and Its Applications, vol. 522, pp. 350-364, 2019.

[28] K. Bina and N. Moghadas, "BIM-ABM simulation for emergency evacuation from conference hall, considering 
gender segregation and architectural design," in Architectural Engineering And Design Management, Taylor \& Francis, Milton, UK, 2020.

[29] Y. Shin, S. Kim, and I. Moon, "Simultaneous evacuation and entrance planning in complex building based on dynamic network flows," Applied Mathematical Modelling, vol. 73, pp. 545-562, 2019.

[30] W. Liao, X. Zheng, L. Cheng, Y. Zhao, Y. Cheng, and Y. Wang, "Layout effects of multi-exit ticket-inspectors on pedestrian evacuation," Safety Science, vol. 70, pp. 1-8, 2014.

[31] H. L. Mu, S. M. Lo, W. G. Song, W. Lv, and J. H. Sun, "Impact of wedge-shaped design for building bottlenecks on evacuation time for efficiency optimization," Simulation, vol. 91, no. 11, pp. 1014-1021, 2015.

[32] Z. Li and W. Xu, "Pedestrian evacuation within limited-space buildings based on different exit design schemes," Safety Science, vol. 124, 2020.

[33] M. Haghani and M. Sarvi, "Simulating pedestrian flow through narrow exits," Physics Letters A, vol. 383, no. 2-3, pp. 110-120, 2019.

[34] T. Zhang, S.-S. Huang, X.-L. Zhang, S.-X. Lu, and C.-H. Li, "Effect of exit location on flow of mice under emergency condition," Chinese Physics B, vol. 28, no. 1, 2019.

[35] S. Cao, L. Fu, and W. Song, "Exit selection and pedestrian movement in a room with two exits under fire emergency," Applied Mathematics and Computation, vol. 332, pp. 136-147, 2018.

[36] W. Lei and C. Tai, "Effect of different staircase and exit layouts on occupant evacuation," Safety Science, vol. 118, pp. 258-263, 2019.

[37] X. Song, J. Sun, H. Xie, Q. Li, Z. Wang, and D. Han, "Characteristic time based social force model improvement and exit assignment strategy for pedestrian evacuation," Physica A: Statistical Mechanics and Its Applications, vol. 505, pp. 530-548, 2018.

[38] Q. Liu, "The effect of dedicated exit on the evacuation of heterogeneous pedestrians," Physica A: Statistical Mechanics and Its Applications, vol. 506, pp. 305-323, 2018.

[39] S. Wolfram, "Statistical mechanics of cellular automata," Reviews of Modern Physics, vol. 55, no. 3, pp. 601-644, 1983.

[40] M. Shuaib and Z. Zainuddin, "Incorporating intelligence into exit choice model for typical evacuation," Sains Malaysiana, vol. 46, no. 10, pp. 1997-2005, 2017.

[41] I. M. Sticco, G. A. Frank, S. Cerrotta, and C. O. Dorso, "Room evacuation through two contiguous exits," Physica A: Statistical Mechanics and Its Applications, vol. 474, pp. 172-185, 2017.

[42] K. Zia and A. Ferscha, "A simulation study of exit choice based on effective throughput of an exit area in a multi-exit evacuation situation," in Proceedings of the 13th Ieee/Acm International Symposium on Distributed Simulation And RealTime Applications, pp. 235-238, Washington, DC, USA, October 2009.

[43] M. Haghani and M. Sarvi, "Simulating dynamics of adaptive exit-choice changing in crowd evacuations: model implementation and behavioural interpretations," Transportation Research Part C: Emerging Technologies, vol. 103, pp. 56-82, 2019.

[44] S. M. Lo, H. C. Huang, P. Wang, and K. K. Yuen, "A game theory based exit selection model for evacuation," Fire Safety Journal, vol. 41, no. 5, pp. 364-369, 2006.

[45] J. Y. Wang, J. Ma, P. Lin et al., "Experimental study of architectural adjustments on pedestrian flow features at bottlenecks," Journal Of Statistical Mechanics-Theory And Experiment, vol. 2019, no. 8, p. 83402, 2019.

[46] N. Shiwakoti, X. Shi, and Z. Ye, "A review on the performance of an obstacle near an exit on pedestrian crowd evacuation," Safety Science, vol. 113, pp. 54-67, 2019.

[47] L. Wu and X. Fan, "Simulation of pedestrian evacuation flow based on different selection strategies," in Proceedings of the IEEE Advanced Information Technology, Electronic and Automation Control Conference (IAEAC), Chongqing, China, December 2015.

[48] H. Yue, B.-Y. Zhang, C.-F. Shao, and Y. Xing, "Exit selection strategy in pedestrian evacuation simulation with multiexits," Chinese Physics B, vol. 23, no. 5, 2014.

[49] Z. Zainuddin and M. M. A. Shuaib, "Modelling the independence factor and its effect on the preferred force of the social force model in emergency and non-emergency situations," Applied Mathematics \& Information Sciences, vol. 5, no. 1, pp. 53-64, 2011.

[50] Z. Zainuddin and M. Shuaib, "Modification of the decisionmaking capability in the social force model for the evacuation process," Transport Theory And Statistical Physics, vol. 39, no. 1, pp. 47-70, 2010.

[51] G. F. Ma and Z. J. Wu, "BIM-based building fire emergency management: combining building users' behavior decisions," Automation in Construction, vol. 109, p. 16, 2020.

[52] M. Lujak and S. Giordani, "Centrality measures for evacuation: finding agile evacuation routes," Future Generation Computer Systems, vol. 83, pp. 401-412, 2018.

[53] W. Song, Y. Yu, and T. Chen, "Influences of exit conditions on pedestrian evacuation," Fire Safety Science, vol. 12, no. 2, pp. 100-104, 2003.

[54] Y. Han, W. Wang, Q. Fan, and Z. Hu, "Optimization of building evacuation exits based on genetic algorithm and pedestrian evacuation model," Computer Engineering and Applications, vol. 56, no. 4, pp. 1-11, 2020.

[55] D. Helbing, J. Keltsch, and P. Molnár, "Modelling the evolution of human trail systems," Nature, vol. 388, no. 6637, pp. 47-50, 1997.

[56] A. Garcimartin, D. R. Parisi, J. M. Pastor, C. Martín-Gómez, and I. Zuriguel, "Flow of pedestrians through narrow doors with different competitiveness," Journal of Statistical Mechanics-Theory And Experiment, vol. 2016, no. 4, p. 43402, 2016.

[57] W. Liao, A. Tordeux, A. Seyfried et al., "Measuring the steady state of pedestrian flow in bottleneck experiments," Physica A: Statistical Mechanics and Its Applications, vol. 461, pp. 248261, 2016.

[58] T. Huan-Huan, D. Li-Yun, and X. Yu, "Influence of the exits' configuration on evacuation process in a room without obstacle," Physica A: Statistical Mechanics and Its Applications, vol. 420, pp. 164-178, 2015.

[59] D. L. Zhao, L. Z. Yang, and J. Li, "Exit dynamics of occupant evacuation in an emergency," Physica A-Statistical Mechanics And Its Applications, vol. 363, no. 2, pp. 501-511, 2006.

[60] Z. Shahhoseini and M. Sarvi, "Traffic flow of merging pedestrian crowds: how architectural design affects collective movement efficiency," Transportation Research Record: Journal of the Transportation Research Board, vol. 2672, no. 20, pp. 121-132, 2018.

[61] Y. Ma, R. K. K. Yuen, and E. W. M. Lee, "Effective leadership for crowd evacuation," Physica A: Statistical Mechanics and Its Applications, vol. 450, pp. 333-341, 2016. 